\title{
Lyapunov-based Optimizing Control of Nonlinear
}

\section{Blending Processes}

\author{
Tor A. Johansen*, Daniel Sbárbaro** \\ *Department of Engineering Cybernetics, Norwegian University of Science and Technology, \\ 7491 Trondheim, Norway. \\ **Department of Electrical Engineering, University of Concepción, Concepción, Chile.
}

\begin{abstract}
Blending processes consisting of linear dynamics and a static nonlinearity are considered. We propose a control law that optimizes the equilibrium point of the process and regulates the output to the corresponding equilibrium state. A control-Lyapunov function is used to derive a stable optimizing update law for the equilibrium point, in combination with an LQ feedback law for tracking the optimized equilibrium point. The analysis and design also incorporates the use of an observer for state and bias estimation. Experimental results using a laboratory scale colorant blending process illustrate the efficiency of the method.
\end{abstract}

\section{INTRODUCTION}

Blending processes arise in a wide range of industries, for example gasoline blending [1], [2], [3], [4], food, pharmaceuticals and colorant blending [5], [6], [7]. They are characterized by fairly simple dynamics, essentially transport delays due to piping in addition to linear blending dynamics. However, such processes contain static nonlinearities that describe the multivariable relationship between the composition of the mixture (i.e. the concentrations of its individual substances) and the output variables (the overall quality measures of the mixture). Hence, a blending controller needs to take into account such nonlinearities and couplings in order to achieve a mixture with the given quality specification. A further complication is that the composition of the mixture can usually not be measured directly, so there may be a need for a state estimator or observer if a full state feedback approach is taken. Optimization of the use of raw materials is also desirable, especially if the number of feed components are larger than the number of output variables, which is the more common situation.

In this work we develop and analyze an optimization-based control technique for such nonlinear blending processes, which we assume are described by a linear time-invariant state-space model plus a static output nonlinearity. The idea is based on a control Lyapunov function (CLF) that is augmented with a steady-state optimization criterion that ensures that the quality measure of the mixture is according to specification, the cost of raw materials is minimized, and a barrier function that ensures that the 
steady-state operating point does not violate any feed pump constraints. Based on this CLF and a dynamic control specification, an asymptotically optimizing controller is derived similar to CLF design of adaptive and nonlinear controllers [8]. After this basic control strategy is developed, we extend the controller with an observer that takes into account that the state is not measured, and there are unknown output disturbances. Stability, convergence and asymptotic optimality of the closed loop is then proved under general conditions. The use of the suggested method is illustrated using a laboratory scale colorant blending process where three colorants are blended to give a required color, for example specified in terms of its RGB values.

The present paper extends preliminary results [9] with the introduction and analysis of an observer in the closed loop, and experimental results. The idea of seeking only asymptotic optimality is similar to [10] and the sub-optimal approach considered in [11]. The approach provides an alternative control method for systems of the Wiener class, as treated recently in [12], [13], [14]. Extremum seeking approaches (see [15] and the references therein) are similar in spirit but relies on direct measurement of the quality variables, or at least an uncertain parameterized quality measure [16]. A distinct difference compared to the present approach is that extremum seeking does not assume explicit knowledge of a nonlinear model that relates the states to the quality measures. The general idea of combining steady-state optimization with dynamic control is well known in the process control literature, e.g. [17]. Nonlinear PID controllers are commonly used in industrial blending applications. The present work has the advantage that it allows explicit optimization of quality versus cost, and analysis of stability in the presence of nonlinearities.

\section{A CLASS OF NONLINEAR BLENDING PROCESSES}

In this paper an optimization-based nonlinear controller is developed and analyzed within a theoretical framework in sections III and IV. The nonlinear class of blending processes is written in the state-space form

$$
\begin{aligned}
\dot{x} & =A x+B u \\
y & =g(x)
\end{aligned}
$$

where the state vector $x \in \mathbb{R}^{n}$ typically represents compositions, the input vector $u \in \mathbb{R}^{m}$ represents inlet flow rates, velocities or actuator settings, and the output vector $y \in \mathbb{R}^{p}$ represents the quality measures that specify the control objective. It is generally assumed that there are no more outputs than inputs, i.e. $m \geq p$. The output function $g$ describes how the quality measure $y$ depends in a nonlinear manner on the states under static conditions. The only assumption made on this known nonlinearity is that it is a continuously differentiable nonlinear function.

The controllable and open-loop stable dynamics $(A, B)$ describe flow and blending phenomena. If pumps or valves at the inlet are characterized by monotonous nonlinearities, the validity of the linear dynamics will rely on an linearization of these nonlinearities, either locally or globally by an inverse mapping being used to define the control inputs $u$. 


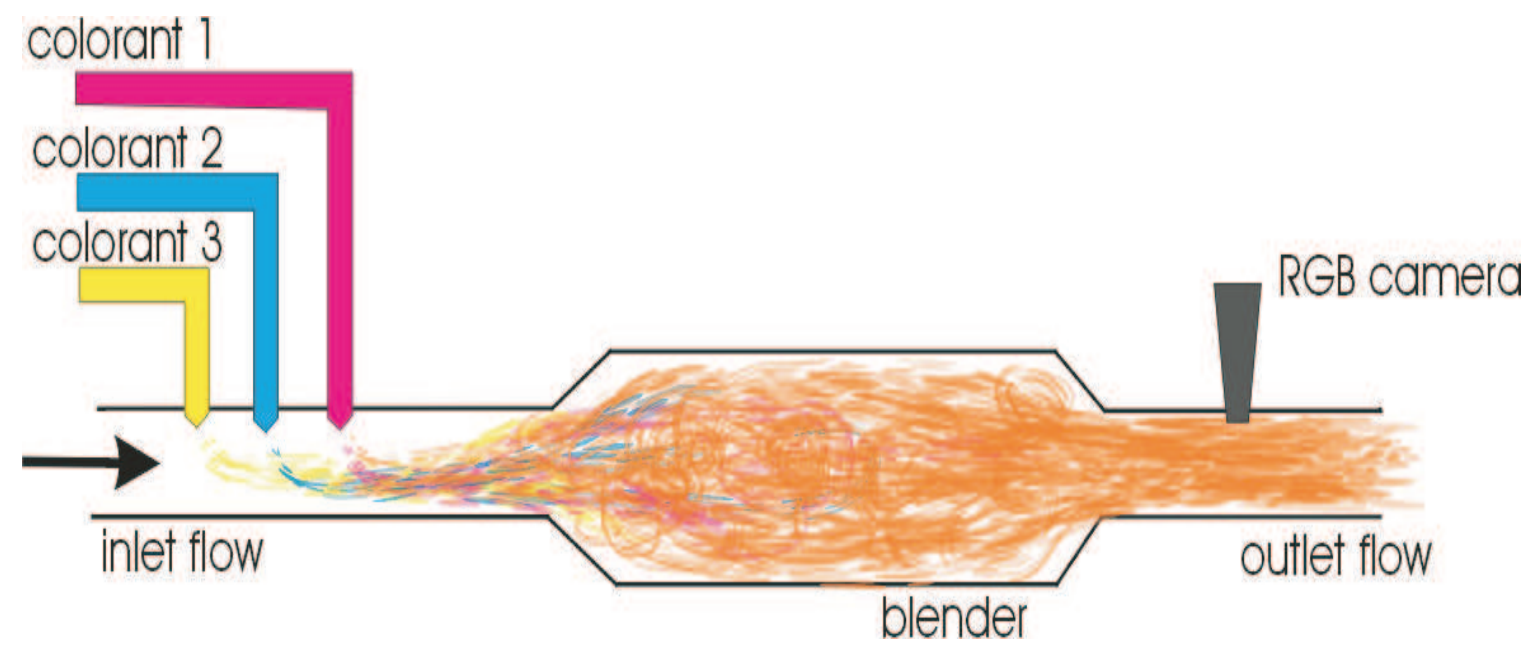

Fig. 1. Colorant blending process.

\section{A. Example: Colorant blending process}

Before we describe the control approach, we describe an experimental (laboratory scale) colorant blending process that will be used to illustrate the method, see Figure 1. The inlet flow contains water from the water supply. The flow rate may be controlled, but is subject to disturbances caused by variations in the supply pressure due to load transients. The inlet flow is mixed with three different colorants, whose flow rate can be controlled via the speeds of individual pumps. The blender unit contains baffles that leads to a non-stationary flow pattern that gives good spatial mixing. At the outlet of the blender there is an RGB camera and an illumination source, confined in a closed environment, that provides a measurement of the color of the mixture. The RGB image is processed to determine an average RGB value for the mixture at the outlet.

Let $u \in \mathbb{R}^{3}$ denote the colorant pump speeds, $z \in \mathbb{R}^{3}$ denote the concentration of the three colorants at the camera position, and $y \in \mathbb{R}^{3}$ denote the measured RGB values ( $y_{1}$ is red, $y_{2}$ is green, and $y_{3}$ is blue). Experiments show that the dynamic response from each colorant pump speed to the corresponding concentration at the blender outlet can be accurately described by a first order linear system with dead-time

$$
\frac{z_{i}}{u_{i}}(s)=\frac{e^{-3.5 s}}{1+3 s}
$$

At steady-state $z_{i}=u_{i}$, and we remark that in this transfer function the units of $z_{i}$ are non-physical units for colorant concentration since they are identical to the units for the pump speed. The steady-state mapping $y=g(u)$ from pump speeds to RGB values can be described theoretically by the Kubelka-Munk theory and a sensor model [18]. However, since the parameters of these models are not easily determined, we propose a parameterized nonlinear mapping described by polynomials of the form:

$$
y_{i}=a_{i 1} u_{1}+a_{i 2} u_{2}+a_{i 3} u_{3}+a_{i 4} u_{1}^{2}+a_{i 5} u_{2}^{2}+a_{i 6} u_{3}^{2}+a_{i 7} u_{1} u_{2}+a_{i 8} u_{1} u_{3}+a_{i 9} u_{2} u_{3}+a_{i 10}
$$

for $i=1,2,3$. Appendix A describes model identification and validation results. We remark that the quadratic model is not globally valid, but valid within a sufficient range for the experiments presented here. 
For the purpose of the analysis, the time-delay can be assumed to be approximated with a finite-dimensional linear time-invariant system incorporated in (1). In the experimental implementation used with the laboratory process, a discrete-time formulation is used which can directly incorporate the time-delay, see section V.

\section{StATE FEEDBACK OPtIMIZING CONTROLLER}

Let $\theta \in \mathbb{R}^{m}$ denote the equilibrium input, to be optimized, and $\xi$ be the corresponding equilibrium state for (1) such that

$$
0=A \xi+B \theta
$$

It follows that there exists a matrix $F$ such that $\xi=F \theta$. The control strategy is to employ a linear state feedback where the reference and feed-forward are defined from the equilibrium input $\theta$ that is optimized such that the equilibrium output equals a given reference value $g(F \theta)=y^{*}$. In other words, the objective is to choose $\theta$ such that it minimizes the steady-state performance criterion

$$
J\left(\theta ; y^{*}\right)=\frac{1}{2}\left(y^{*}-g(F \theta)\right)^{T} W\left(y^{*}-g(F \theta)\right)
$$

where $W>0$ is a symmetric weighting matrix. In general, there may be many solutions $\theta$ for a given $y^{*}$, so this criterion may also include the cost of raw materials

$$
J_{d}\left(\theta ; y^{*}\right)=\frac{1}{2}\left(y^{*}-g(F \theta)\right)^{T} W\left(y^{*}-g(F \theta)\right)+d^{T} \theta
$$

there $d \in \mathbb{R}^{m}$ is a constant vector such that $d_{i}>0$ represents the unit cost of raw material with index $i$. With the laboratory colorant blending process used as an example in this paper there are no additional degrees of freedom (only 3 colorants) such that it is sufficient to use (6) and we refer to [9] for a simulation example considering the redundant situation using (7). Finally, the dynamic performance specification is given in terms of an infinite-horizon LQ criterion with weighting matrices $Q>0$, and $R>0$ :

$$
J_{L Q}(u ; x(t), \theta)=\int_{t}^{\infty}\left((x(\tau)-F \theta)^{T} Q(x(\tau)-F \theta)+(u(\tau)-\theta)^{T} R(u(\tau)-\theta)\right) d \tau
$$

Hence, the controller objective is to simultaneously optimize the equilibrium point and regulate the optimal equilibrium.

Assume a compact and convex constraint set $\Theta_{c} \subset \mathbb{R}^{m}$ is given. Such a convex set can be derived from input constraints such as $u_{\min } \leq u \leq u_{\max }$ which means the equilibrium input $\theta$ must satisfy $u_{\min } \leq \theta \leq u_{\max }$. Let the interior of $\Theta_{c}$ be denoted $\operatorname{int}\left(\Theta_{c}\right)$, its boundary be denoted $\partial \Theta_{c}$ and assume the set $\Theta_{c}$ is represented as

$$
\Theta_{c}=\left\{\theta \in \mathbb{R}^{m} \mid c(\theta) \leq 0\right\}
$$

where $c: \mathbb{R}^{m} \rightarrow \mathbb{R}^{q}$. The set of vectors $\theta \in \Theta_{c}$ satisfying first order optimality conditions is defined in terms of the Karush-KuhnTucker (KKT) conditions [19]:

$$
\Theta_{c}^{*}=\left\{\theta \in \Theta_{c} \mid \nabla_{\theta} J\left(\theta ; y^{*}\right)+\sum_{i=1}^{q} \lambda_{i} \nabla c_{i}(\theta)=0, \lambda_{i} c_{i}(\theta)=0, \lambda_{i} \geq 0\right\}
$$


Define the logarithmic barrier function

$$
b(\theta)=b_{0}-\sum_{i=1}^{q} \log \left(-c_{i}(\theta)\right)
$$

where the constant $b_{0} \in \mathbb{R}$ is selected such that $b(\theta)>0$ for all $\theta \in \operatorname{int}\left(\Theta_{c}\right)$, which will be useful in the Lyapunov analysis that will follow. Such a $b_{0}$ exists due to the compactness of $\Theta_{c}$. A fundamental property of this barrier function is that it is well-defined and convex on $\operatorname{int}\left(\Theta_{c}\right)$ since $\Theta_{c}$ is convex [20]. Moreover, its value goes to infinity as $\theta \rightarrow \partial \Theta_{c}$, and it is undefined outside $\Theta_{c}$. Next, define a CLF

$$
V(x, \theta, \varrho)=(x-F \theta)^{T} P(x-F \theta)+\beta\left(J\left(\theta ; y^{*}\right)+\varrho b(\theta)\right)+\frac{1}{2} \varrho^{2}
$$

where $P>0$ and $\beta>0$ will be specified shortly. For all weighting parameters $\varrho>0$ the barrier function will prevent $\theta(t)$ from escaping the interior of $\Theta_{c}$. When applying such barrier functions in numerical optimization, convergence toward the optimum is usually achieved by letting $\varrho \rightarrow 0$ as $t \rightarrow \infty$, [19], [20], and we take a similar approach here (at least for the theoretical analysis in this section):

$$
\dot{\varrho}=-\nu \varrho, \varrho(0)>0
$$

with $\nu>0$ is a time-scale parameter for the barrier function. To avoid loss of performance these dynamics should be chosen faster than the desired closed loop dynamics, but not too fast in order to avoid numerical difficulties in the implementation. The time-derivative of $V$ along trajectories of the closed loop system is given by

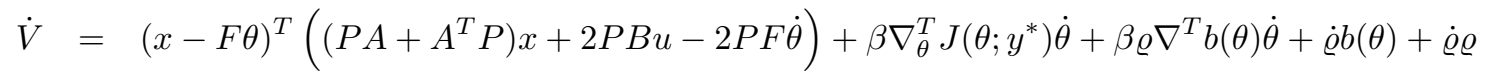

We choose the optimizing dynamic feedback

$$
\dot{\theta}=-\Gamma \ell\left(\theta, \varrho ; y^{*}\right)
$$

with some symmetric $\Gamma>0$ and $\ell\left(\theta, \varrho ; y^{*}\right)=\nabla_{\theta} J\left(\theta ; y^{*}\right)+\varrho \nabla b(\theta)$. The control input is chosen according to the LQ controller

$$
u=-R^{-1} B^{T} P(x-F \theta)+\theta
$$

and the symmetric matrix $P>0$ satisfies the algebraic Riccati equation

$$
A^{T} P+P A-2 P B R^{-1} B^{T} P=-Q
$$

This leads to

$$
\dot{V}=-(x-F \theta)^{T} Q(x-F \theta)-\beta \ell^{T}\left(\theta, \varrho ; y^{*}\right) \Gamma \ell\left(\theta, \varrho ; y^{*}\right)-\nu \varrho b(\theta)-\nu \varrho^{2}+2(x-F \theta)^{T} P F \Gamma \ell\left(\theta, \varrho ; y^{*}\right)
$$

The indefinite term in (18) is dominated by the negative quadratic terms as shown in the proof of the following result: 
Proposition 1: Consider the optimizing controller described above and assume $\Theta_{c}$ is a convex and compact set. Then this controller has the following properties for all $x(0) \in \mathbb{R}^{n}, \theta(0) \in \operatorname{int}\left(\Theta_{c}\right)$ and $\varrho(0)>0$ :

1. If $J$ is strictly convex, then $\Theta_{c}^{*}=\left\{\theta^{*}\right\}$, the equilibrium point $(x, \theta, \varrho)=\left(F \theta^{*}, \theta^{*}, 0\right)$ is asymptotically stable, and $g(x(t)) \rightarrow y^{*}$ as $t \rightarrow \infty$.

2. If $J$ is radially unbounded, then all variables are uniformly bounded and $\theta(t) \in \operatorname{int}\left(\Theta_{c}\right)$ for all $t \geq 0$. Moreover, $\theta(t) \rightarrow \Theta_{c}^{*}$, and $\|x(t)-F \theta(t)\| \rightarrow 0$ as $t \rightarrow \infty$.

Proof. Using Young's inequality $2 a b \leq a^{2} / k+b^{2} k$ for $k>0,(18)$ implies

$$
\dot{V} \leq-\|x-F \theta\|^{2}(\underline{\sigma}(Q)-2 k \bar{\sigma}(P F \Gamma))-\left\|\ell\left(\theta, \varrho ; y^{*}\right)\right\|^{2}(\underline{\sigma}(\Gamma) \beta-2 \bar{\sigma}(P F \Gamma) / k)-\nu \varrho^{2}-\nu \varrho b(\theta)
$$

The first term is made negative by choosing the arbitrary constant $k>0$ sufficiently small. The second term is made negative by choosing the arbitrary constant $\beta>0$ sufficiently large. Notice that $\varrho(t)=\varrho(0) \exp (-\beta t)$ such that $\varrho(t)>0$ for all $t \geq 0$. Since $b(\theta)$ goes unbounded and all other terms remain bounded when $\theta$ approaches the boundary of $\Theta_{c}$, it is clear that $\theta(t) \in \operatorname{int}\left(\Theta_{c}\right)$ for all $t \geq 0$. Hence, the last term in (19) is non-positive because $b(\theta) \geq 0$ for all $\theta \in \operatorname{int}\left(\Theta_{c}\right)$. It follows immediately that $\dot{V}(t) \leq 0$ for all $t \geq 0$ and $\|\varrho(t) b(\theta(t))\|,\|x(t)-F \theta(t)\|,|\varrho(t)|$ and $\left\|\ell\left(\theta(t), \varrho(t) ; y^{*}\right)\right\|$ are uniformly bounded.

In part $1, \theta^{*}$ is a unique global minimum due to strict convexity, and the parameter $b_{0}$ is chosen such that $V\left(F \theta^{*}, \theta^{*}, 0\right)=0$. We have

$$
\begin{aligned}
E & =\left\{(x, \theta, \varrho) \in \mathbb{R}^{n} \times \Theta_{c} \times[0, \infty) \mid \dot{V}(x, \theta, \varrho)=0\right\} \\
& =\left\{(x, \theta, \varrho) \in \mathbb{R}^{n} \times \Theta_{c} \times[0, \infty) \mid \varrho=0, \varrho b(\theta)=0, x=F \theta, \nabla_{\theta} J\left(\theta ; y^{*}\right)+\varrho \nabla b(\theta)=0\right\}
\end{aligned}
$$

Elementary calculations show

$$
\nabla b(\theta)=-\sum_{i=1}^{q} \frac{\nabla c_{i}(\theta)}{c_{i}(\theta)}
$$

Define the vector $\lambda \in \mathbb{R}^{q}$ in terms of its components (which can be interpreted as Lagrange multipliers, see also [19]):

$$
\lambda_{i}=-\frac{\varrho}{c_{i}(\theta)}
$$

which is well-defined since $\theta \in \operatorname{int}\left(\Theta_{c}\right)$. Hence, the last condition in (20) can be written

$$
\ell\left(\theta, \varrho ; y^{*}\right)=\nabla_{\theta} J\left(\theta ; y^{*}\right)+\lambda^{T} \nabla c(\theta)=0
$$

Since $\varrho \geq 0$ and $c(\theta)<0$ for all $\theta \in \operatorname{int}\left(\Theta_{c}\right)$, it follows that $\lambda \geq 0$. Hence, the 1 st and 3rd KKT condition in (10) are satisfied. Since $\varrho=0$ the 2 nd KKT condition is also satisfied due to (22), and we conclude that $\theta=\theta^{*}$ in (20). It follows that $E=\left\{\left(\theta^{*}, \xi^{*}, 0\right)\right\}$ and part 1 of the proposition is proven by Barbashin-LaSalle's theorem [21].

Part 2 can be proven as follows. Since $b$ is locally Lipschitz in $\operatorname{int}\left(\Theta_{c}\right)$ the conditions of Barbalat's lemma hold [21], and we 
conclude that $\dot{V}(t) \rightarrow 0$ as $t \rightarrow \infty$ such that (19) implies

$$
\begin{aligned}
\varrho(t) & \rightarrow 0, & & \text { as } \quad t \rightarrow \infty \\
\varrho(t) b(\theta(t)) & \rightarrow 0, & & \text { as } \quad t \rightarrow \infty \\
\|x(t)-F \theta(t)\| & \rightarrow 0, & & \text { as } \quad t \rightarrow \infty \\
\left\|\nabla_{\theta} J\left(\theta(t) ; y^{*}\right)+\varrho(t) \nabla b(\theta(t))\right\| & \rightarrow 0, & & \text { as } \quad t \rightarrow \infty
\end{aligned}
$$

As above, with $\lambda_{i}(t)=-\varrho(t) / c_{i}(\theta(t))$, it is clear that (27) implies

$$
\nabla_{\theta} J\left(\theta(t) ; y^{*}\right)+\sum_{i=1}^{q} \lambda_{i}(t) \nabla c_{i}(\theta(t)) \rightarrow 0, \quad \text { as } \quad t \rightarrow \infty
$$

Because all functions are continuous in $\operatorname{int}\left(\Theta_{c}\right)$ and (24) implies $\lambda_{i}(t) c_{i}(\theta(t)) \rightarrow 0$ as $t \rightarrow \infty$ for all $i \in\{1,2, \ldots, q\}$, it is evident that all KKT conditions in (10) hold asymptotically. Since $\lambda_{i}(t)>0$ for all $t \geq 0$, we conclude $\theta(t) \rightarrow \Theta_{c}^{*}$ as $t \rightarrow \infty$.

With constant gain matrix $\Gamma>0$, eq. (15) corresponds to a gradient descent minimization. Notice, however, that $\Gamma$ in (15) can be replaced by any time- or state-dependent positive definite-matrix, such as a possibly modified inverse Hessian of $J$, leading to a Newton-method, in order to improve speed of convergence [19].

In a discrete-time implementation, a line search method is useful to adapt the gain such that descent and convergence are guaranteed. Consider two consecutive sampling instants $t_{k}$ and $t_{k+1}$. A discrete-time implementation of (15) is then

$$
\theta\left(t_{k+1}\right)=\theta\left(t_{k}\right)+\left(t_{k+1}-t_{k}\right) a\left(t_{k}\right) \Gamma \ell\left(\theta\left(t_{k}\right), \varrho\left(t_{k}\right) ; y^{*}\right)
$$

where $a\left(t_{k}\right) \in(0,1]$ is the line search parameter that must be chosen such that $V\left(t_{k+1}\right) \leq V\left(t_{k}\right)$. Several line search strategies are available [19]. In the experiments described in section (V) we utilized 29 with $a\left(t_{k}\right)=1$ as a default initial value, and iterations $a\left(t_{k}\right) \leftarrow a\left(t_{k}\right) / 3$ until descent of the Lyapunov function was achieved.

Global optimality is implicit in part 1 of Proposition 1, while only local convergence is ensured in part 2. To increase the chance of converging to a global minimum it is possible to search for values of $\theta$ that gives negative jumps in $J\left(\theta ; y^{*}\right)$, if the value of this function seems to converge to a sub-optimal value. The algorithm can then be extended with a resetting mechanism that resets the value of $\theta(t)$ to such a more promising value, if the optimization seems to get stuck in a local minimum.

\section{OBSERVER-BASED OPTIMIZING CONTROLLER}

The CLF design of the controller and optimizer in section III does not take into account that the state cannot in general be measured. Furthermore, feed pumps, supply pressure, feed compositions and the output map $g$ may be subject to uncertainty. In order to avoid steady-state error due to this uncertainty it is necessary with feedback from the measurement $y$. This is achieved through an observer that estimates the state $x$ and the output bias $e$, defined by the equation $y=g(x)+e$, and a controller that compensates for this bias. We therefore describe a closely related observer-based optimizing controller that utilizes the Internal 


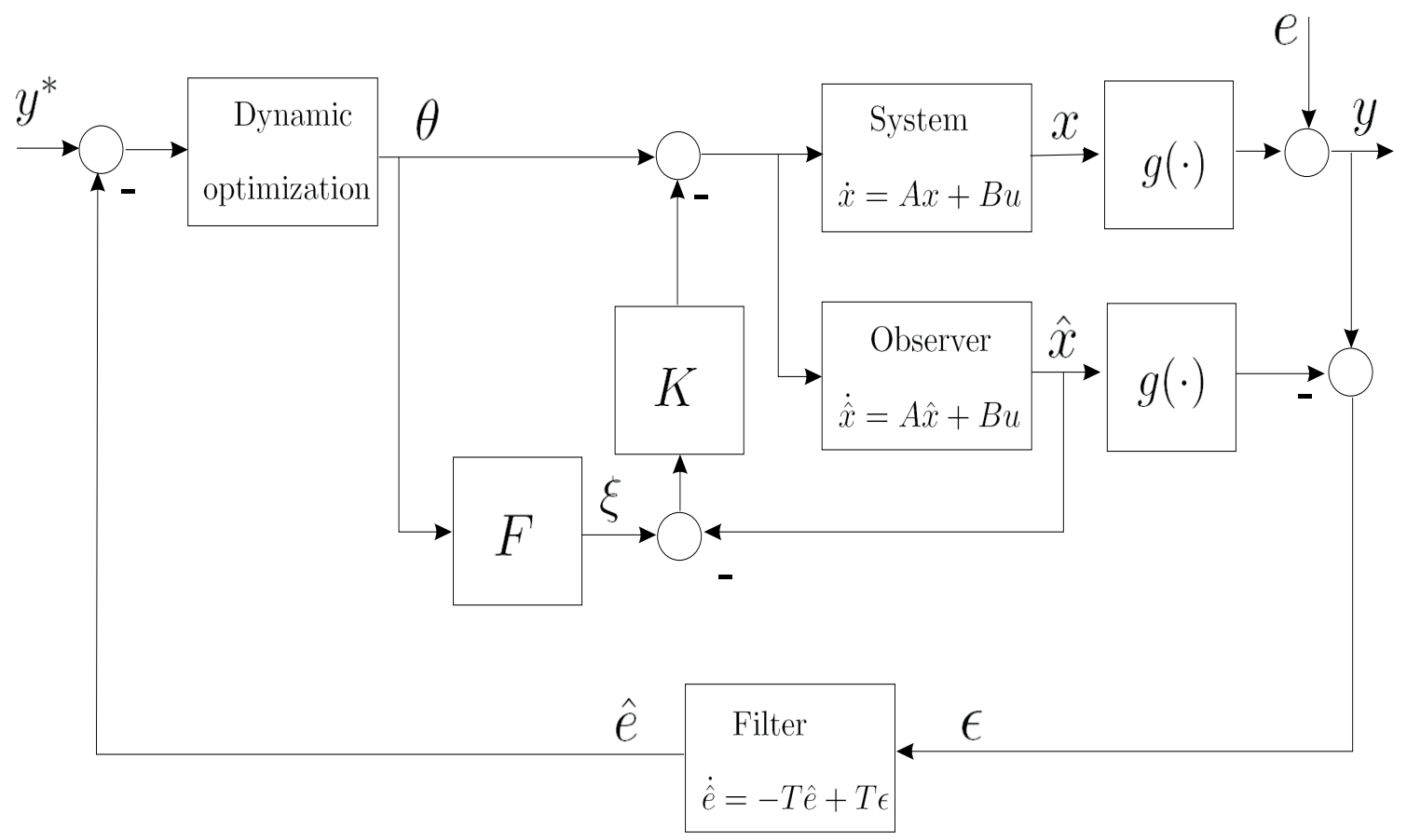

Fig. 2. Observer-based optimizing controller for the nonlinear blending process.

Model Control (IMC) principle [22] and shows that the observer cannot destabilize the control system. Without loss of generality, we neglect the constraints in the analysis in this section for simplicity. Since the blending process is in general open loop stable, we employ an open-loop observer for $x$. Assuming the bias $e$ is an unknown constant and $y^{*}$ is a constant output reference, the closed loop system illustrated in Figure 2 containing the plant, observer, controller and dynamic optimization is described by the following set of differential and algebraic equations

$$
\begin{aligned}
\dot{x} & =A x+B u \\
y & =g(x)+e \\
\dot{\hat{x}} & =A \hat{x}+B u \\
\dot{\hat{e}} & =-T \hat{e}+T(y-g(\hat{x})) \\
\dot{\theta} & =-\Gamma \nabla_{\theta} J\left(\theta, y^{*}-\hat{e}\right) \\
u & =-K(F \theta-\hat{x})+\theta
\end{aligned}
$$

where $K=R^{-1} B^{T} P$. The observer gains are tuned via the diagonal matrix $T>0$. The following result shows that the observer and control errors converge under mild conditions:

Proposition 2: Suppose $A$ is Hurwitz, $g$ is Lipschitz, and there exist a constant $\kappa_{1}>0$ such that $\nabla g(x) F F^{T} \nabla^{T} g(x) \geq \kappa_{1} I$. Then for any initial conditions all signals are bounded, and the trajectories of the closed loop system (30)-(35) converges such that $\|\hat{x}(t)-x(t)\| \rightarrow 0, \hat{e}(t) \rightarrow e, \theta(t) \rightarrow \Theta^{*},\|x(t)-F \theta(t)\| \rightarrow 0$, and $y(t) \rightarrow y^{*}$ as $t \rightarrow \infty$. 
Proof: We assume without loss of generality in this proof that $p=m$. Consider the following Lyapunov function candidate

$$
V(x, \hat{x}, \theta, e)=(\hat{x}-F \theta)^{T} P(\hat{x}-F \theta)+\beta J\left(\theta, y^{*}-\hat{e}\right)+(x-\hat{x})^{T} \Lambda(x-\hat{x})+(e-\hat{e})^{T} S(e-\hat{e})
$$

where $\beta<0$ and symmetric matrices $P>0, S>0$ and $\Lambda>0$ will be specified later. Differentiating with respect to time along trajectories of the closed loop system gives

$$
\begin{aligned}
\dot{V}= & 2(\dot{\hat{x}}-F \dot{\theta})^{T} P(\hat{x}-F \theta)+\beta \nabla_{\theta}^{T} J\left(\theta, y^{*}-\hat{e}\right) \dot{\theta}-\beta \nabla_{y^{*}}^{T} J\left(\theta, y^{*}-\hat{e}\right) \dot{\hat{e}}+2(\dot{x}-\dot{\hat{x}})^{T} \Lambda(x-\hat{x})+2(\dot{e}-\dot{\hat{e}})^{T} S(e-\hat{e}) \\
= & 2((A+B K)(\hat{x}-F \theta)-F \dot{\theta})^{T} P(\hat{x}-F \theta)-\beta \dot{\theta}^{T} \Gamma^{-1} \dot{\theta}+\beta \nabla_{y^{*}}^{T} J\left(\theta, y^{*}-\hat{e}\right)(T \hat{e}-T(g(x)+e-g(\hat{x}))) \\
& +(x-\hat{x})^{T}\left(A^{T} \Lambda+\Lambda A\right)(x-\hat{x})-(e-\hat{e})^{T}\left(T^{T} S+S T\right)(e-\hat{e}+g(x)-g(\hat{x})) \\
= & -(\hat{x}-F \theta) Q(\hat{x}-F \theta)-2 \dot{\theta}^{T} F^{T} P(\hat{x}-F \theta)-\beta \dot{\theta}^{T} \Gamma^{-1} \dot{\theta}-\beta \nabla_{y^{*}}^{T} J\left(\theta, y^{*}-\hat{e}\right) T(e-\hat{e}) \\
& -\beta \nabla_{y^{*}}^{T} J\left(\theta, y^{*}-\hat{e}\right) T(g(x)-g(\hat{x}))-(e-\hat{e})^{T}\left(T^{T} S+S T\right)(g(x)-g(\hat{x})) \\
& +(x-\hat{x})^{T}\left(A^{T} \Lambda+\Lambda A\right)(x-\hat{x})-(e-\hat{e})^{T}\left(T^{T} S+S T\right)(e-\hat{e})
\end{aligned}
$$

where $P>0$ satisfies the Lyapunov equation

$$
(A+B K)^{T} P+P(A+B K)=-Q
$$

for some arbitrary $Q>0$. Recall that

$$
\begin{aligned}
\dot{\theta}=-\Gamma \nabla_{\theta} J\left(\theta, y^{*}-\hat{e}\right) & =\Gamma(\nabla g(F \theta) F)^{T} W\left(y^{*}-\hat{e}-g(F \theta)\right) \\
& =\Gamma(\nabla g(F \theta) F)^{T} \nabla_{y^{*}} J\left(\theta, y^{*}-\hat{e}\right)
\end{aligned}
$$

Hence,

$$
\nabla_{y^{*}} J\left(\theta, y^{*}-\hat{e}\right)=-\left(F^{T} \nabla^{T} g(F \theta)\right)^{-1} \Gamma^{-1} \dot{\theta}
$$

Using the multi-variable version of Taylor's theorem, [23], we have $g(x)-g(\hat{x})=\nabla g(\tilde{x})(x-\hat{x})$ for some $\tilde{x}$ on the line segment between $x$ and $\hat{x}$. We now define

$$
\mathbb{Y}(x, \hat{x}, \theta)=-\frac{1}{2} T\left(F^{T} \nabla^{T} g(F \theta)\right)^{-1} \Gamma^{-1}
$$

such that $\dot{V}$ can be written in the following form

$$
\begin{aligned}
\dot{V}= & -(\hat{x}-F \theta) Q(\hat{x}-F \theta)-\beta \dot{\theta}^{T} \Gamma^{-1} \dot{\theta}+(x-\hat{x})^{T}\left(A^{T} \Lambda+\Lambda A\right)(x-\hat{x})-(e-\hat{e})^{T}\left(T^{T} S+S T\right)(e-\hat{e}) \\
& -2 \dot{\theta}^{T} F^{T} P(\hat{x}-F \theta)+2 \beta \dot{\theta}^{T} \mathbb{Y}^{T} \nabla g(\tilde{x})(x-\hat{x})+2 \beta \dot{\theta}^{T} \mathbb{Y}^{T}(e-\hat{e})+(e-\hat{e})^{T}\left(T^{T} S+S T\right) \nabla g(\tilde{x})(x-\hat{x}) \\
\leq & -\|\hat{x}-F \theta\|^{2} \underline{\sigma}(Q)-\|\dot{\theta}\|^{2} \beta \underline{\sigma}\left(\Gamma^{-1}\right)-\|x-\hat{x}\|^{2} \underline{\sigma}\left(-A^{T} \Lambda-\Lambda A\right)-\|e-\hat{e}\|^{2} \underline{\sigma}\left(T^{T} S+S T\right) \\
& +2\|\dot{\theta}\| \cdot\|\hat{x}-F \theta\| \bar{\sigma}\left(F^{T} P\right)+2\|\dot{\theta}\| \cdot\|x-\hat{x}\| \beta L_{g} \bar{\sigma}(\mathbb{Y})+2\|\dot{\theta}\| \cdot\|e-\hat{e}\| \beta \bar{\sigma}(\mathbb{Y}) \\
& +2\|e-\hat{e}\| \cdot\|x-\hat{x}\| \bar{\sigma}(S T) L_{g}
\end{aligned}
$$


where $L_{g}$ is the Lipschitz constant for $g$. Using Young's inequality $2 a b \leq a^{2} / k+b^{2} k$, where $k>0$ is arbitrary, gives

$$
\begin{aligned}
\dot{V} \leq & -\|\hat{x}-F \theta\|^{2}\left(\underline{\sigma}(Q)-\bar{\sigma}\left(F^{T} P\right) \mu_{1}\right) \\
& -\|\dot{\theta}\|^{2}\left(\beta \underline{\sigma}\left(\Gamma^{-1}\right)-\bar{\sigma}\left(F^{T} P\right) / \mu_{1}-\beta L_{g} \bar{\sigma}(\mathbb{Y}) \mu_{2}-\beta \bar{\sigma}(\mathbb{Y}) \mu_{2}\right) \\
& -\|x-\hat{x}\|^{2}\left(\underline{\sigma}\left(-A^{T} \Lambda-\Lambda A\right)-\beta L_{g} \bar{\sigma}(\mathbb{Y}) / \mu_{2}-L_{g} \bar{\sigma}(S T)\right) \\
& \left.-\|e-\hat{e}\|^{2}\left(\underline{\sigma}\left(T^{T} S+S T\right)-\beta \bar{\sigma}(\mathbb{Y}) / \mu_{2}-L_{g} \bar{\sigma}(S T)\right)\right)
\end{aligned}
$$

where $\mu_{1}, \mu_{2}>0$ are arbitrary. At this point, recall that in addition to $\mu_{1}$ and $\mu_{2}$, one can choose freely the following parameters that arise in the analysis only (their values have no implications or relevance for the control design or tuning): $\Lambda>0, S>0$, and $\beta>0$. We proceed as follows:

1. Choose $\mu_{1}$ sufficiently small such that $\underline{\sigma}(Q)-\bar{\sigma}\left(F^{T} P\right) \mu_{1}>0$.

2. Choose $\mu_{2}$ sufficiently small and $\beta$ sufficiently large such that $\beta \underline{\sigma}\left(\Gamma^{-1}\right)-\bar{\sigma}\left(F^{T} P\right) / \mu_{1}-\beta L_{g} \bar{\sigma}(\mathbb{Y}) \mu_{2}-\beta \bar{\sigma}(\mathbb{Y}) \mu_{2}>0$. This is in general possible because $\mathbb{Y}$ is bounded.

3. Choose $S$, satisfying the Lyapunov inequality $T^{T} S+S T>0$ (recall that $T>0$ ), so large that $\underline{\sigma}\left(T^{T} S+S T\right)-\beta \bar{\sigma}(\mathbb{Y}) / \mu_{2}-$ $\bar{\sigma}(S T) L_{g}>0$.

4. Choose $\Lambda$ sufficiently large such that $\underline{\sigma}\left(-A^{T} \Lambda-\Lambda A\right)-\beta L_{g} \bar{\sigma}(\mathbb{Y}) / \mu_{2}-\bar{\sigma}(S T) L_{g}>0$.

It follows that $\dot{V}(t) \leq 0$ for all $t \geq 0$ such that all signals are bounded, and Barbalat's lemma immediately gives convergence. Notice that $\dot{\theta}(t) \rightarrow 0$ implies $\nabla_{\theta} J\left(\theta(t), y^{*}-\hat{e}(t)\right) \rightarrow 0$ such that $\theta(t) \rightarrow \Theta^{*}$ as $t \rightarrow \infty$.

We remark that the assumptions are reasonable: $A$ is Hurwitz for blending processes in general. The assumption on positive definiteness of $\nabla g(x) F F^{T} \nabla^{T} g(x)$ and $g$ being Lipschitz is verified to hold for the output map for the colorant process given in section II-A for all states of interest.

We also mention that the filter time constant (diagonal matrix) $T>0$ can be replaced by a time-varying diagonal matrix $T(t) \geq T_{\min }>0$ without affecting the convergence results.

\section{EXPERIMENTAL RESULTS}

The implementation of the controller has three interesting differences compared to the theory outlined in sections III and IV.

- First, a discrete-time model is used in the observer, such that the dead-time can be implemented exactly

$$
z_{i}(k+8)=0.8465 z_{i}(k+7)+0.1535 u_{i}(k)
$$

The sampling interval is $T_{s}=0.5 \mathrm{sec}$, and the model (46) contains additional states for the 7 sample dead time.

- Second, a dead-time compensation scheme similar to [24] is employed. This means that we apply feedback from the observed non-delayed state $\hat{z}(k)$ when computing $u(k)$. From a theoretical point of view this essentially corresponds to a partial state 
feedback through the gain matrix $K$ and will trivially stabilize the plant. In a continuous-time analogy, Proposition 2 still holds, since open loop stability of the plant implies existence of a $P>0$ in the proof.

- Finally, we have chosen the barrier function weight $\varrho(t)=0.25$, rather than letting it go to zero asymptotically. This is done in order to avoid numerical problems, and its only consequence is that the input constraints will hold with some insignificant small margin.

The tuning of the controller is done as follows. The feedback gain matrix $K$ is chosen via an LQ design (based on a discrete-time state-space model without time-delay since the feedback is from the observed non-delayed states). The design parameters are tuned experimentally to maximize the bandwidth with a sufficient degree of robustness. The IMC filter time constant $T$ is replaced by a rate-limiter (the theory still holds for any time-varying $T(t)>0$ ) in order not to introduce unnecessary phase loss. The optimizer gain $\Gamma(t)=\gamma(t) I$ is chosen as $\gamma(t)=2.5$, but the value is reduced, if necessary, in a line search phase to achieve descent as described at the end of section III. This is a simple choice that can be replaced by more advanced methods, but does not seem to limit the performance of the control system in our case. The pumps' speed are limited at the minimum value $60 \mathrm{rpm}$, and the maximum value $260 \mathrm{rpm}$. The weighting matrix is $W=I$.
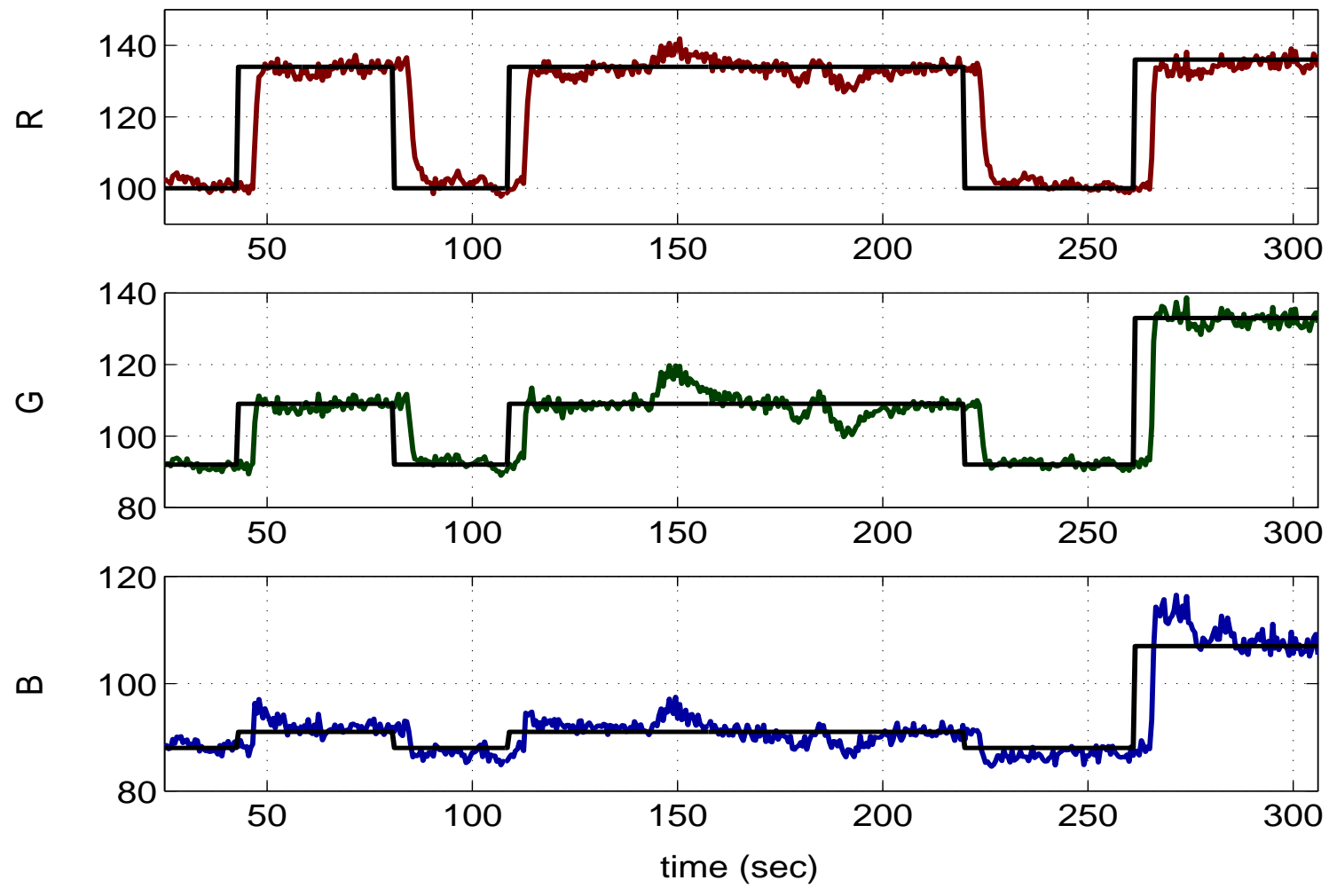

Fig. 3. Experiment 1, optimizing controller: RGB values (measured and reference).

Experimental results are shown in Figures 3 - 5. The RGB reference makes several simultaneous step changes throughout the experiment, and we observe that there is accurate tracking and disturbance rejection. The asymptotic optimization converges quickly, and imposes no significant loss of performance. The bias estimates show that the model of the static nonlinearity is far 

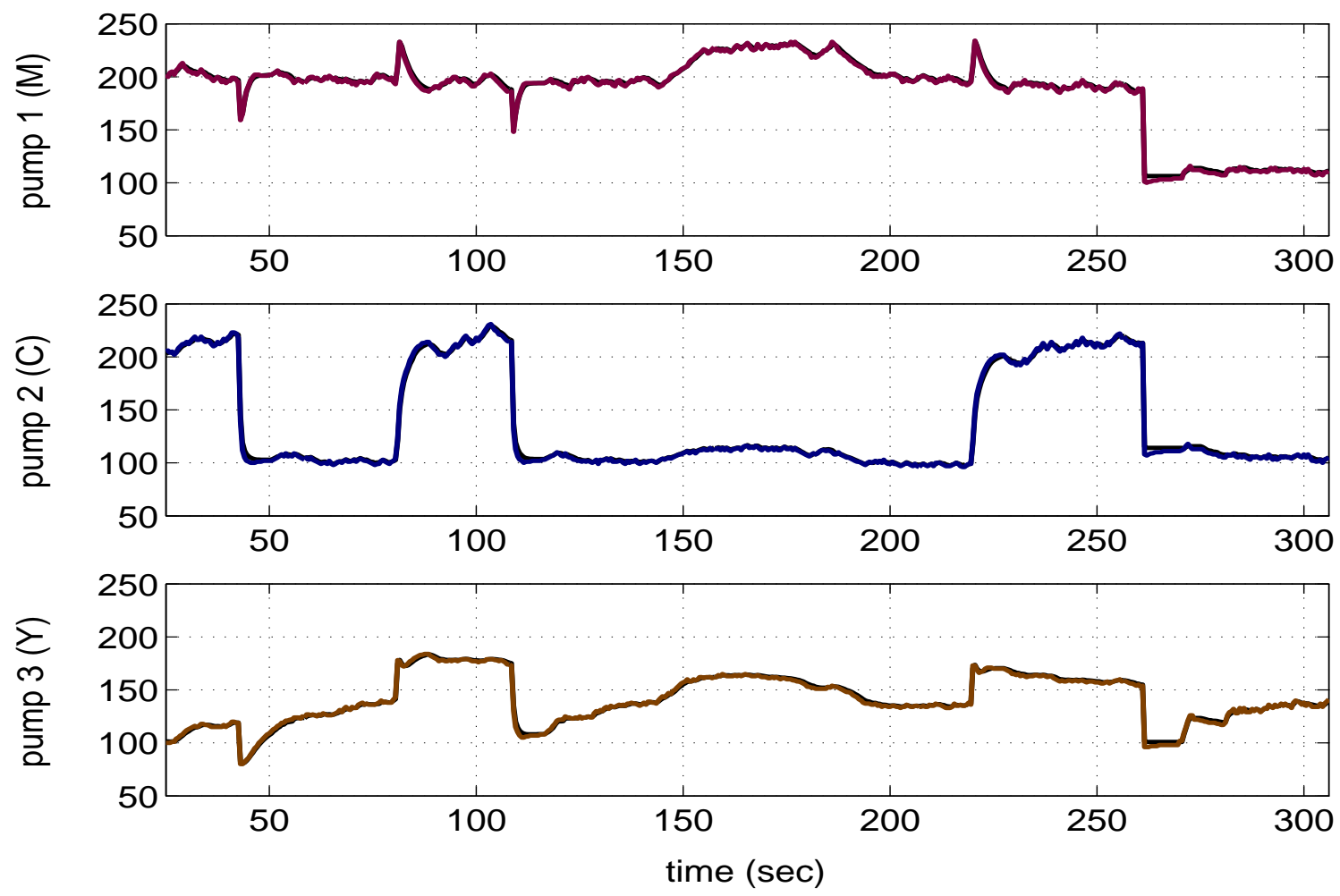

Fig. 4. Experiment 1, optimizing controller: Optimized pump speed values (rpm).

\begin{tabular}{cccc}
\hline Controller & $\mathrm{R}$ & $\mathrm{G}$ & $\mathrm{B}$ \\
\hline Optimizing & 2.2163 & 1.5939 & 0.9553 \\
PI & 2.3061 & 2.9493 & 2.4581 \\
\hline
\end{tabular}

TABLE I

Averaged Integrated Absolute Error (IAE)

from an ideal model, yet the bias estimation scheme works well and avoids steady-state tracking error. At time $t=140 \mathrm{sec}$ a $25 \%$ increase in water supply flow rate occurs, leading to a significant disturbance on the process. The bias estimator detects the resulting output bias (see Figure 5) and the disturbance only leads to a fairly small transient on the RGB output. At time $t=185$ sec the water supply flow rate goes back to its original value, and another small transient results. The results with the proposed controller can be compared with the use of three well-tuned SISO PI-controllers, cf. Figures 6 - 7 and Appendix B for a description of the tuning. The PI-controllers lead to sluggish response because they must be tuned somewhat conservatively due to the static nonlinearities, and they do not account for all couplings in the plant. This is confirmed by the Integrated Absolute Error (IAE) measures reported in table I. 

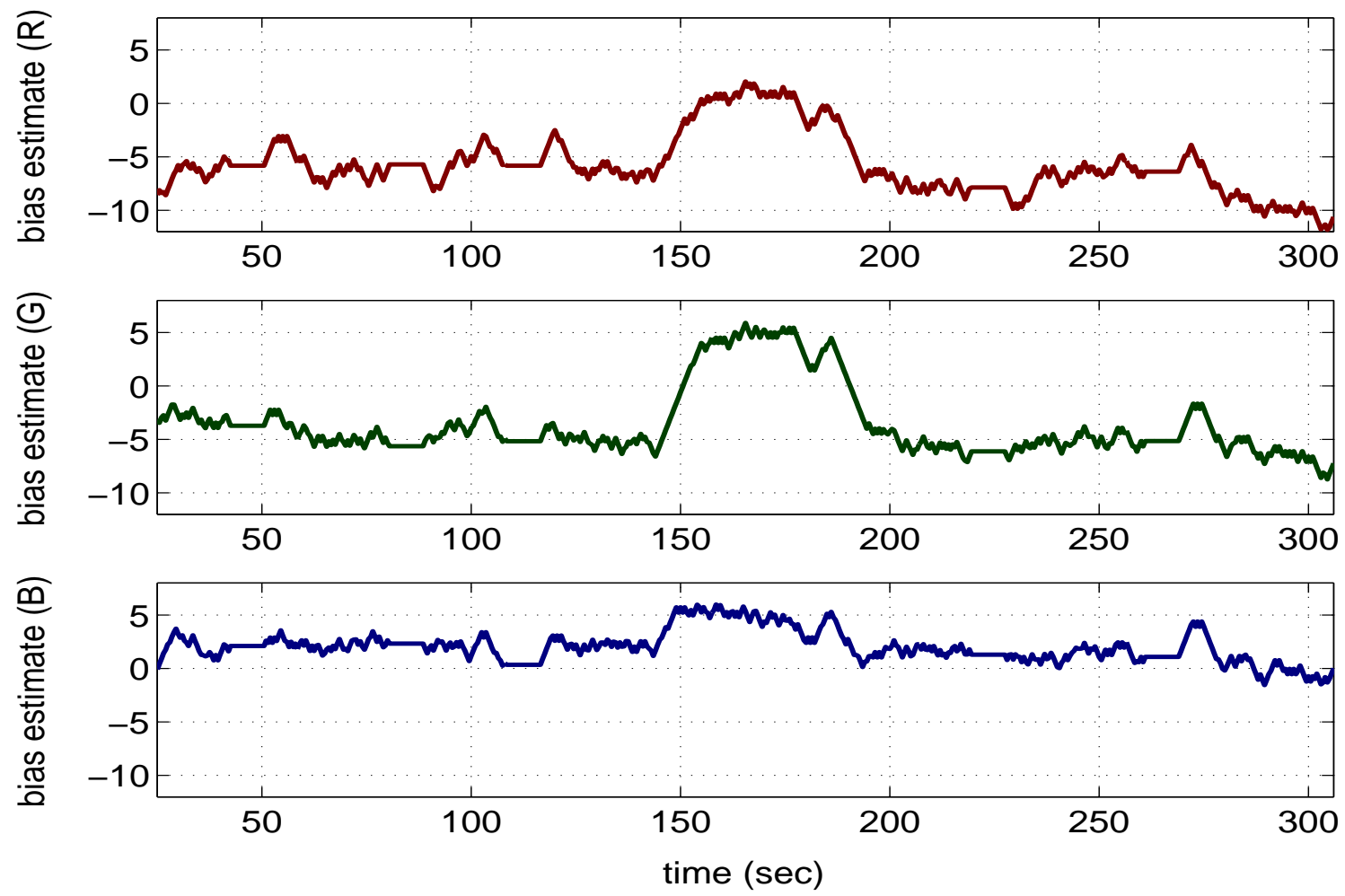

Fig. 5. Experiment 1, optimizing controller: Bias estimates

\section{CONCLUSIONS}

A nonlinear optimizing controller for blending processes is developed using a CLF approach, essentially by augmenting a CLF with an objective function for the parameter $\theta$ that characterizes the equilibrium point. These results also hold in the case of having an observer that takes into account the effect of the uncertainty and time delay associated to the real plant. Real-time experiments have illustrated the feasibility and good performance of the proposed approach. The CLF design idea is borrowed from the area of nonlinear and adaptive control [8] where $\theta$ usually denotes unknown parameters to be adapted.

\section{APPENDIX A: COLORANT BLENDING PROCESS IDENTIFICATION AND VALIDATION}

In this section we describe how the laboratory blending process model was identified and validated. The input was a sequence covering the range between 40 and $280 \mathrm{rev} / \mathrm{min}$, see Figure 8 . The parameters used in the experiments are identified from measured data using a standard least squares method, and given in Table II.

For model validation, we have used another set of data collected in a different day. The identified nonlinear model provides a reasonable approximation, as seen in figure 9. In order to illustrate the process nonlinear characteristics, the performance of an identified linear model, considering only the linear terms in equation (4), is depicted in figure 10. 

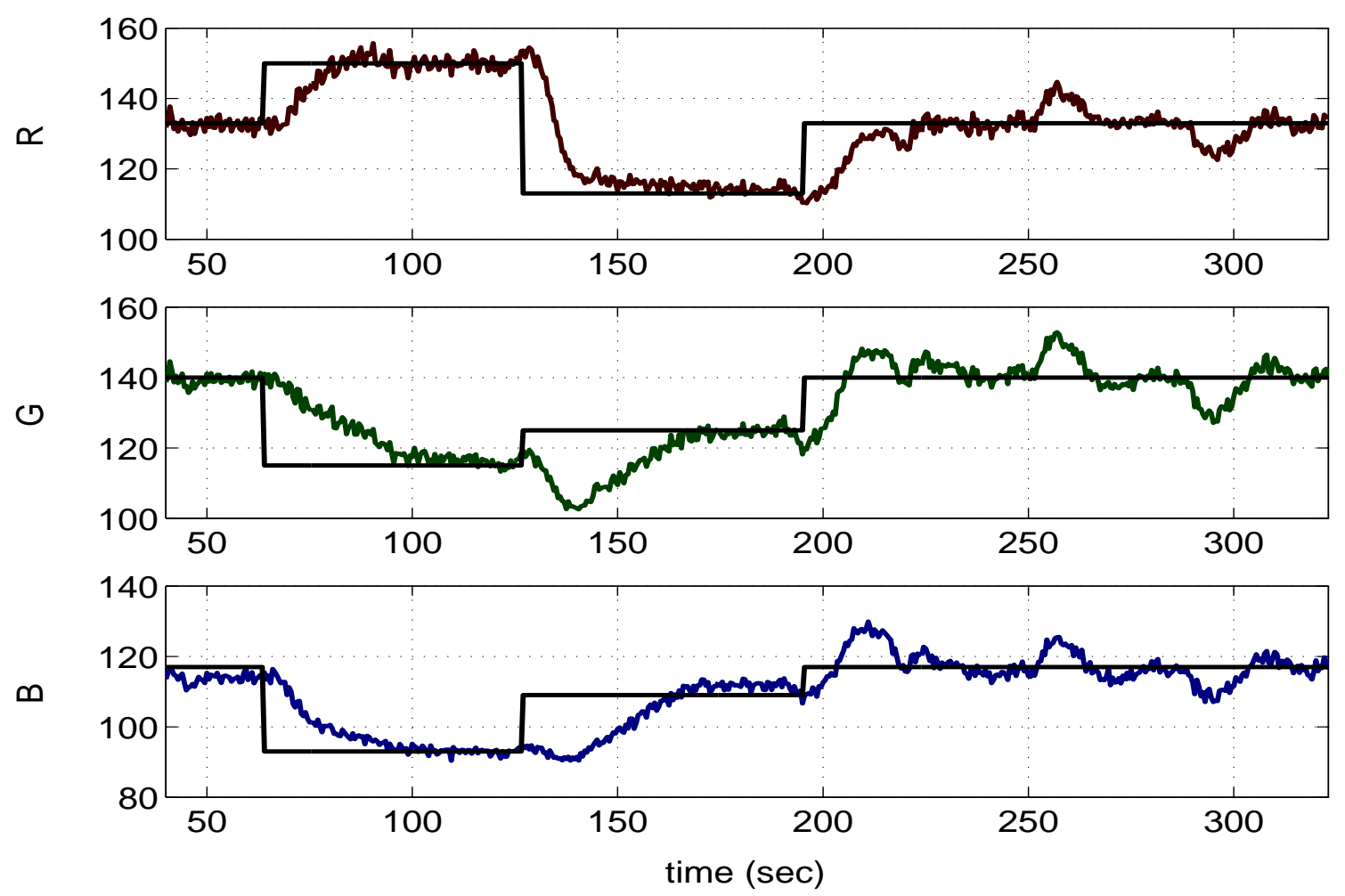

Fig. 6. Experiment 2, PI-controller: RGB values (measured and reference).

\section{APPENDIX B: TUNING THE PI CONTROLLER}

In order to tune the conventional PI SISO controllers, we carried out the following procedure. Firstly, a set of simple first order plus delay models, between the pump speeds and each color channel, were identified from step responses around an operating point, see Figure 11. These experiments gave as a result the following gain matrix:

$$
G(0)=\left[\begin{array}{ccc}
-0.14 & -0.5 & -0.52 \\
-0.62 & -0.62 & 0 \\
0 & & -0.18
\end{array}\right],
$$

and a time-constant delay ratio of about 0.8 for all transfer functions. Secondly, the pairing of the 3 SISO controllers was performed using the Relative Gain Array (RGA) :

$$
\Lambda=\left[\begin{array}{ccc}
-0.3899 & 1.3899 & 0 \\
1.3899 & -0.3899 & 0 \\
0 & 0 & 1
\end{array}\right]
$$

This simple analysis suggests to control the red channel with pump 2, the green one with pump 1 , and the blue one with pump 3. Finally, the tuning of PI controllers was carried out by using initially the IMC tuning rules [22], but since the model is only accurate around one operating point, the parameters have to be detuned in order to have an acceptable response in other regions. Figures 6 - 7 illustrates the dynamical behavior obtained. Notice the different response character exhibited by the closed loop 

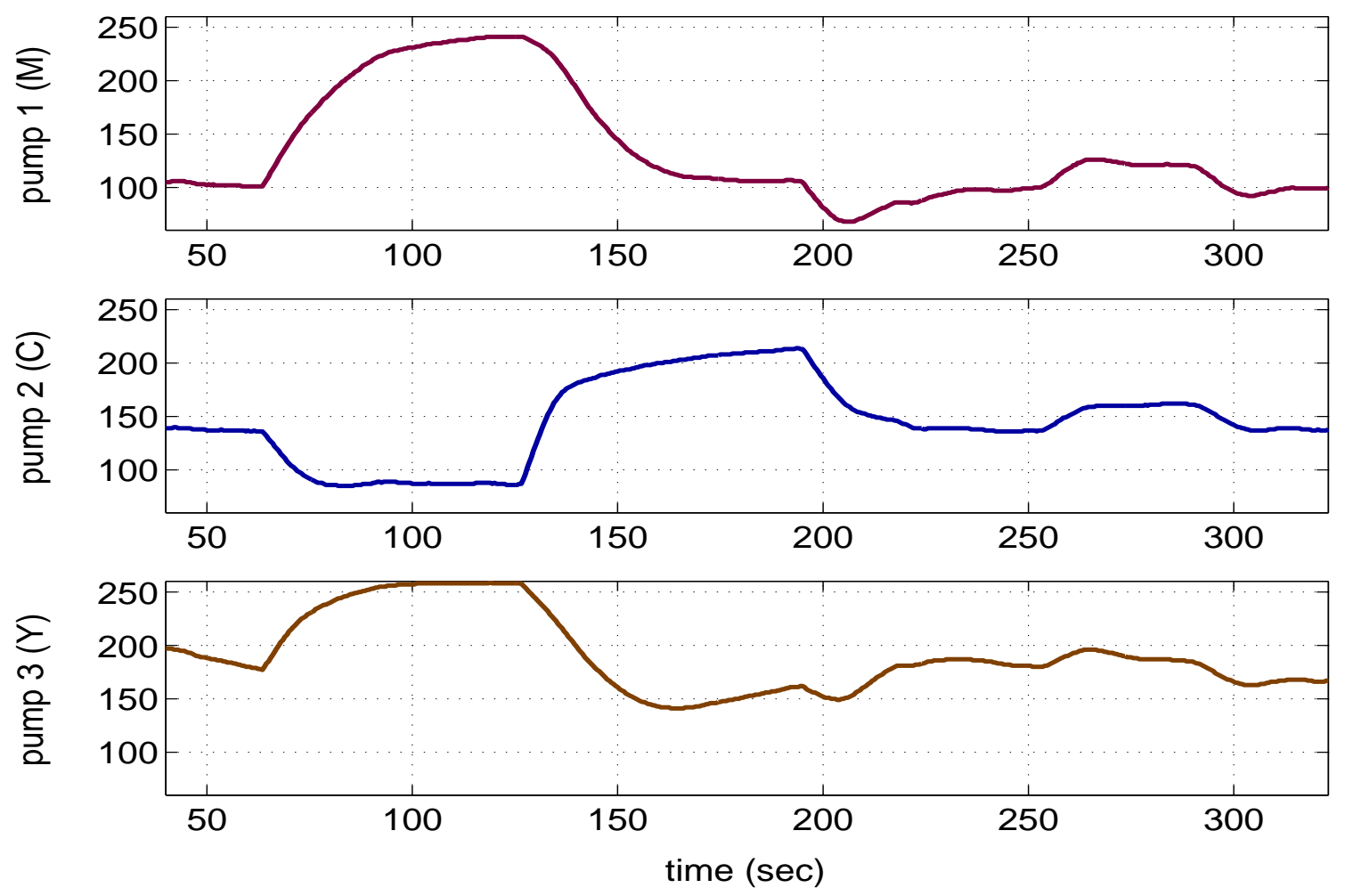

Fig. 7. Experiment 2, PI-controller: Pump speed values (rpm).

system, it is possible to see initially over-damped response, and then non minimum-phase behaviors. The PI-controllers lead to this sluggish responses because they must be tuned somewhat conservatively due to the nonlinearities, and they lead to significantly larger transients because they do not account for all the coupling in the plant.

\section{REFERENCES}

[1] A. Diaz and J.A. Barsamian, "Meet changing fuel requirements with online blend optimization,” Hydrocarbon Processing, , no. 2, pp. 71-76, 1996.

[2] A. Singh, J. F. Forbes, P. Vermeer, and S.S. Woo, "Model-based real-time optimization of automotive gasoline blending operations," J. Process Control, vol. 10 , pp. 43-58, 2000.

[3] J. Alvarez-Ramirez, A. Morales, and R. Suarez, "Robustness of a class of bias update controllers for blending systems," Ind. Eng. Chem. Res., vol. 41, pp. 4786-4793, 2002.

[4] M. Huzmezan, G.A. Dumont, W. A. Gough, T. Janiewicz, S. Kovac, and D. Meade, "A multivariable Laguerre-based indirect adaptive predictive controller applied to a fuel blending process," in AdConIP, Kumamoto, Japan, 2002.

[5] A. Haurani, O. Taha, H. Michalska, and B. Boulet, "Multivariable control of a paper coloring process: A case of study," in American Control Conference, Arlington, VA, June, 2001.

[6] S. Chen, T. Murphy, and R. Subbarayan, "A color measurement and control system for paper-making process," in IEEE Int. Conference on Control applications, Dearborn, MI, September, 1996.

[7] P. R. Belanger, "Linear-programming approach to color-recipe formulation,” Journal of the Optical Society of America, vol. 64, no. 11, pp. 1541-1544, 1974.

[8] M. Krstic, I. Kanellakopoulos, and P. Kokotovic, Nonlinear and Adaptive Control Design, Wiley and Sons, 1995.

[9] T. A. Johansen and D. Sbárbaro, “Optimizing control of over-actuated linear systems with nonlinear output maps via control Lyapunov functions," in European Control Conference, Cambridge, UK, 2003. 

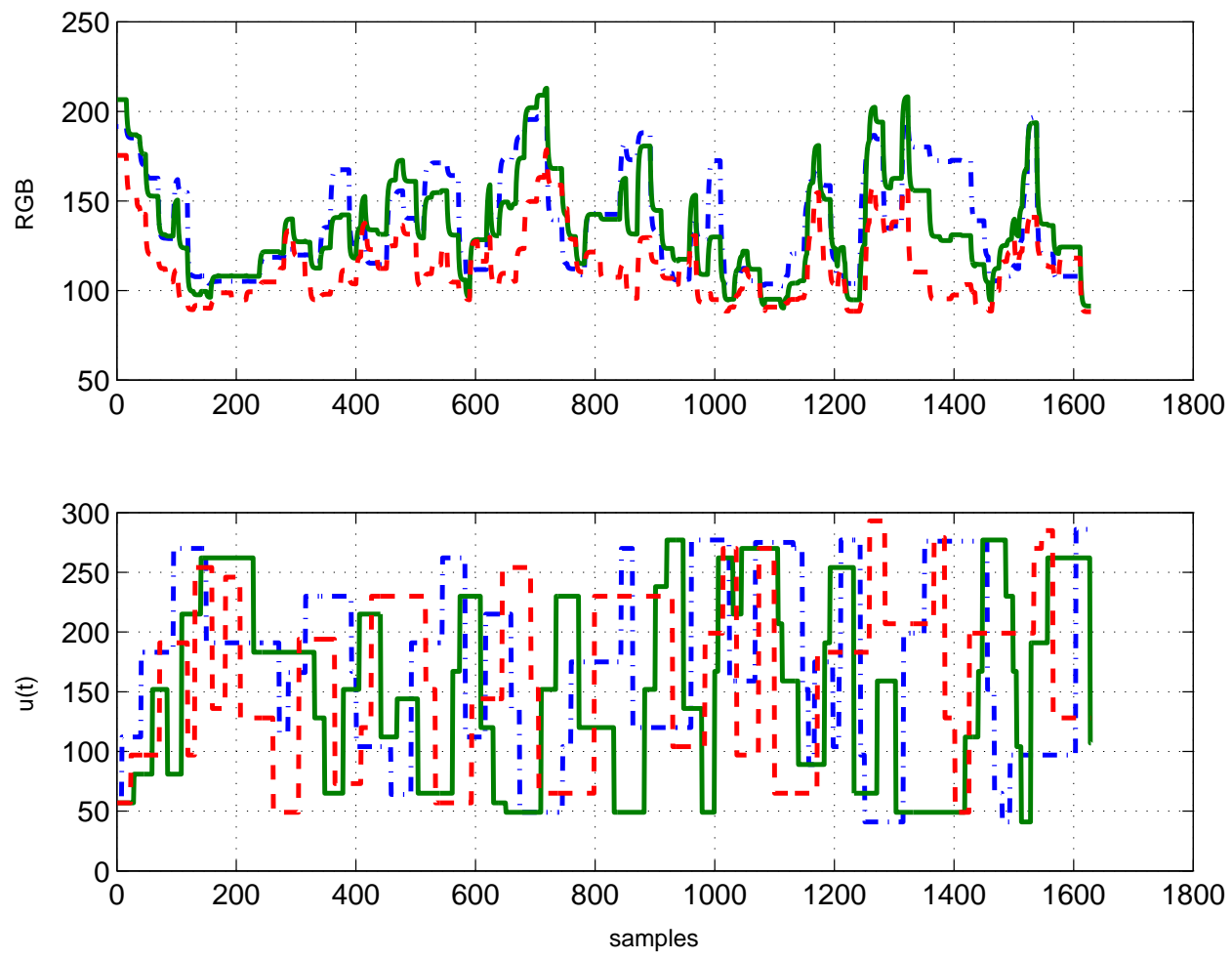

Fig. 8. Data sequence used for identification

[10] M. Cannon and B. Kouvaritakis, "Efficient constrained model predictive control with asymptotic optimality," in Proc. IEEE Conf. Decision and Control, Las Vegas, 2002, pp. ThA12-1.

[11] P. O. M. Scokaert, D. Q. Mayne, and J. B. Rawlings, "Suboptimal model predictive control (feasibility implies stability)," IEEE Trans. Automatic Control, vol. 44, pp. 648-654, 1999.

[12] B.-G. Jeong, K.-Y. Yoo, and Hyun-Ku Rhee, "Nonlinear model predictive control using a Wiener model of a continuous MMA polymerization reactor," Ind. Eng. Chem. Res., vol. 40, pp. 5968-5977, 2001.

[13] H. H. J. Bloemen, C. T. Chou, T. J. J. van den Boom, V. Verdult, M. Verhaegen, and T. C. Backx, "Wiener model identification and predictive control for dual composition control of a distillation column," Journal of Process Control, vol. 11, pp. 601-620, 2001.

[14] S.J. Norquay, A. Palazoglu, and J.A. Romagnoli, "Application of Wiener model predictive control (WMPC) to pH neutralization experiment," IEEE Trans. Control Systems Technology, vol. 7, pp. 437-445, 1999.

[15] M. Krstic and H. H. Wang, "Stability of extremum seeking feedback for general dynamic systems," Automatica, vol. 36, pp. 595-600, 2000.

[16] M. Guay and T. Zhang, “Adaptive extremum seeking control of nonlinear dynamic systems with parametric uncertainties,” Automatica, vol. 39, pp. 12831293, 2003.

[17] S. Skogestad, "Self-optimizing control: the missing link between steady-state optimization and control," Computers and Chemical Engineering, vol. 24, pp. $569-575,2000$.

[18] E. Allen, "Basic equations used in computer color matching," J. Optical Society America, vol. 56, pp. 1256-1259, 1960.

[19] J. Nocedal and S. J. Wright, Numerical Optimization, Springer-Verlag, New York, 1999.

[20] A. V. Fiacco and G. P. McCormick, Nonlinear programming: Sequential unconstrained minimization techniques, J. Wiley and sons, New York, 1968.

[21] H. K. Khalil, Nonlinear Systems, Prentice Hall, 2002.

[22] M. Morari and E. Zafiriou, Robust process control, Prentice-Hall, Englewood Cliffs, NJ, 1989.

[23] R. Abrahamson, J. E. Marsden, and T. Ratiu, Manifolds, Tensor Analysis and Applications (2nd Edition), Springer-Verlag, New York, 1988.

[24] C. Kravaris and R. A. Wright, "Deadtime compensation for nonlinear processes," AIChE Journal, vol. 35, pp. 1535-1542, 1989. 


\begin{tabular}{cccc}
\hline & $a_{1 j}$ & $a_{2 j}$ & $a_{3 j}$ \\
\hline$a_{i 1}$ & -0.168597 & -0.617673 & -0.703415 \\
$a_{i 2}$ & -0.815173 & -0.593565 & -0.236830 \\
$a_{i 3}$ & 0.159001 & 0.192853 & -0.097820 \\
$a_{i 4}$ & 0.000437 & 0.001010 & 0.001358 \\
$a_{i 5}$ & 0.001442 & 0.000853 & 0.000256 \\
$a_{i 6}$ & -0.000106 & -0.000342 & 0.000067 \\
$a_{i 7}$ & 0.000331 & 0.000723 & 0.000512 \\
$a_{i 8}$ & -0.000652 & -0.000609 & 0.000092 \\
$a_{i 9}$ & -0.000074 & 0.000137 & 0.000181 \\
$a_{i 10}$ & 216.92 & 228.17 & 193.38 \\
\hline & & TABLE II &
\end{tabular}

ESTIMATED PARAMETERS OF STATIC NONLINEARITY IN THE LABORATORY COLORANT BLENDING PROCESS.
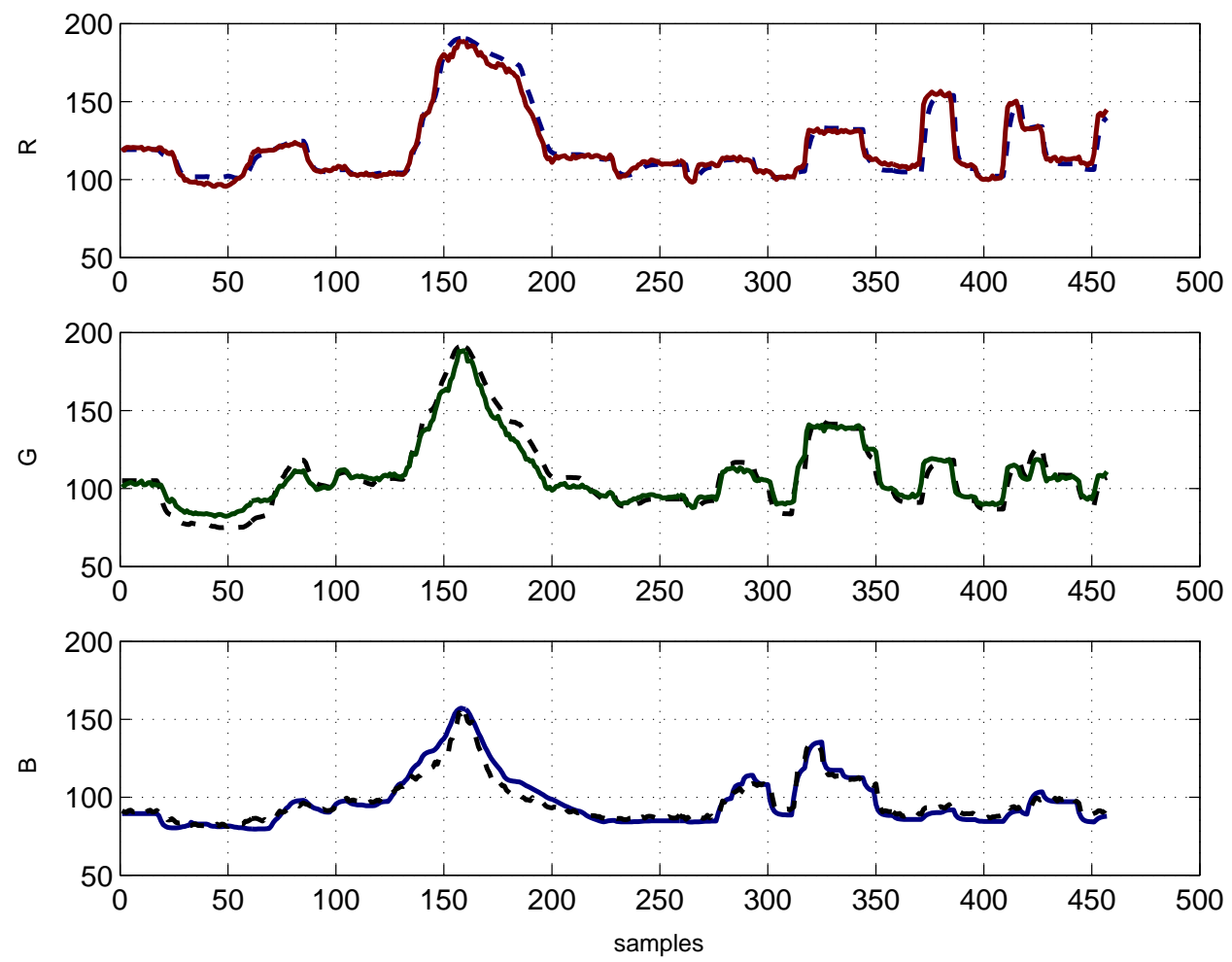

Fig. 9. Validation of the nonlinear system. Dashed curves are experimental data, and solid curves are validation simulations. 
$\simeq$

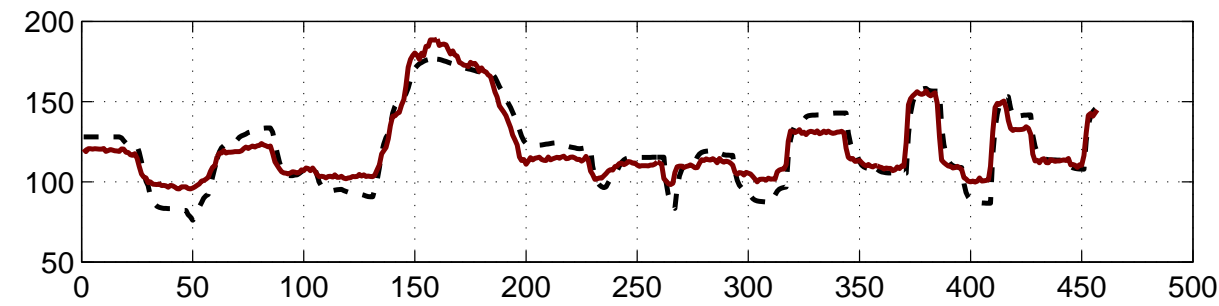

(1)

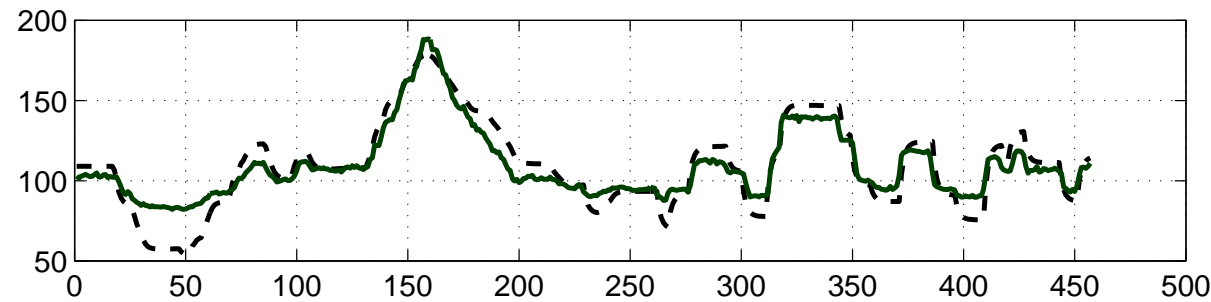

$\infty$

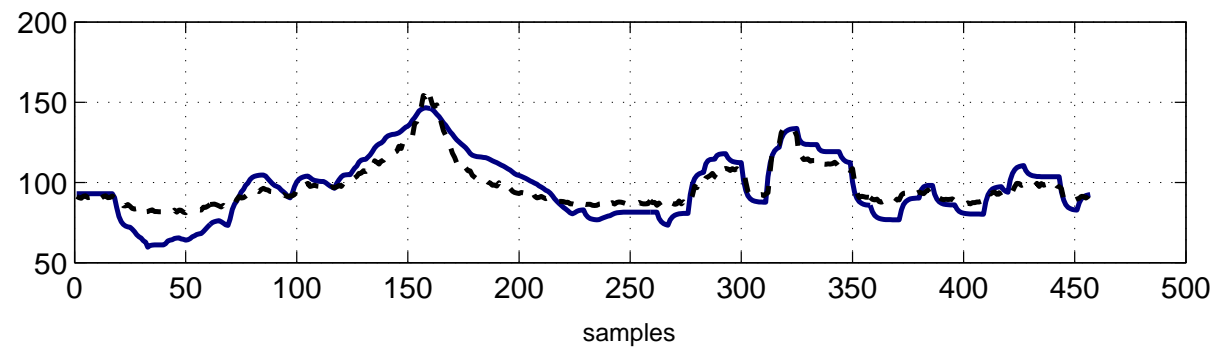

Fig. 10. Validation linear model. Dashed curves are experimental data, and solid curves are validation simulations.
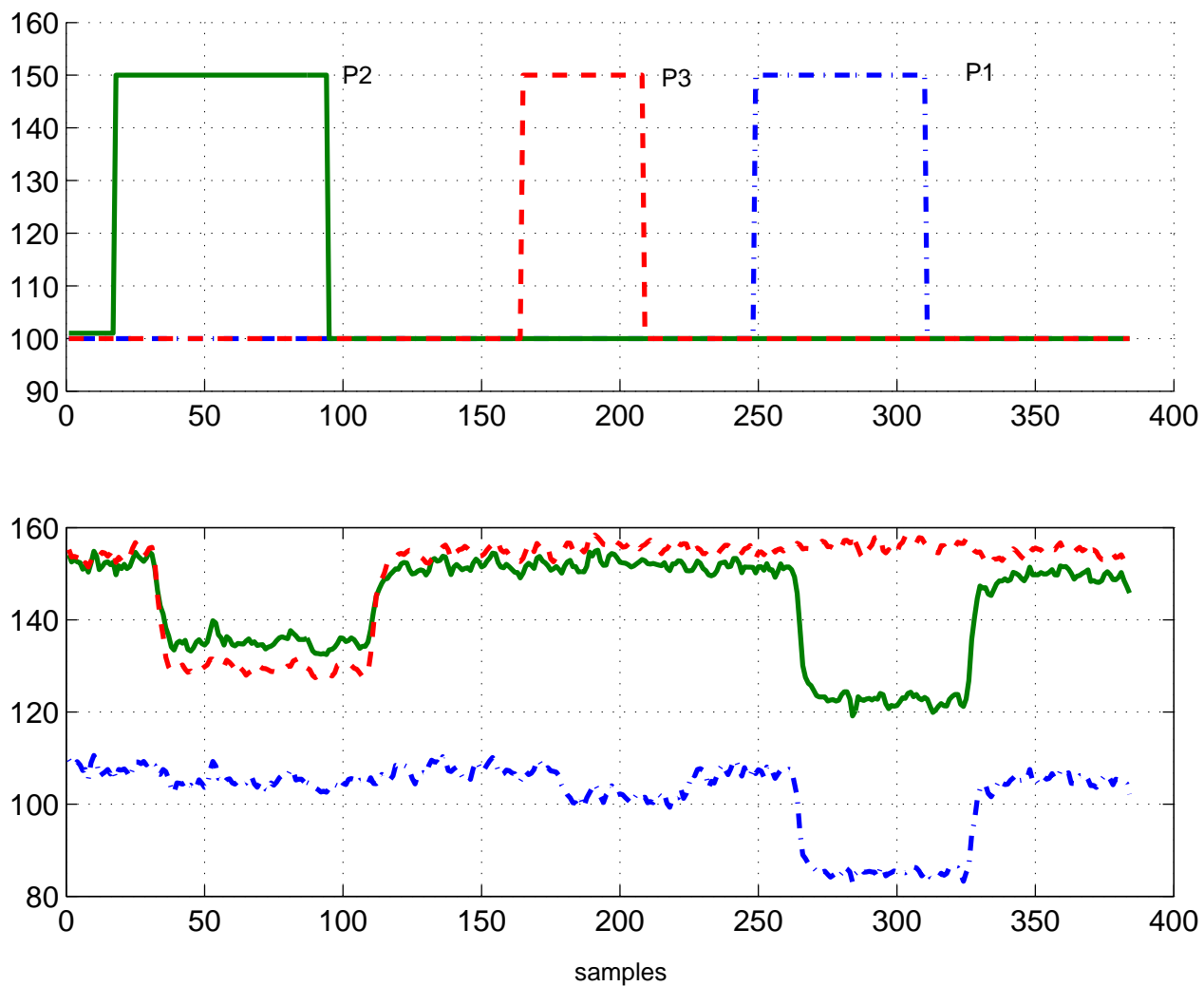

Fig. 11. step responses for the identification of simple linear models. 